\title{
High Speed, High Throughput Two Dimensional Direct Electron Detector Based on the Concept of pnCCDs
}

\author{
L. Strüder ${ }^{1}$, J. Soltau ${ }^{2}$, J. Schmidt ${ }^{1}$, R. Hartmann ${ }^{1}$, M. Huth ${ }^{1}$, H. Soltau ${ }^{2}$, P. Holl ${ }^{1}$, M. Simson ${ }^{2}$, G. Lutz ${ }^{1}$ \\ and H. Ryll ${ }^{1}$
}

${ }^{1}$ PNSensor GmbH, 80803 München, Römerstr. 28, Germany

2 PNDetector GmbH, Emil-Nolde-Str. 10, 81735 München, Germany

Two dimensional direct detection electron imaging solid state sensor systems, subject to intense radiation, suffer from charge overflow to neighbouring pixels. This not only affects the dynamic range and linearity, but also the position precision of e.g. the electron impinging on the detector. Various techniques have been proposed to overcome the problem of charge overflowing the pixels. Several sensors are able to cope with up to a few million signal charges per pixel. In many scientific applications, e.g. in free electron laser science and electron detection in TEMs, this is by far not sufficient. We have developed a technique to handle 1 billion signal charges per pixel without charge spilling over to neighbouring pixel within one readout frame of typically $1 \mathrm{~ms}$. The quality of the point spread function remains unchanged but amplitude information may be degraded. We call this mode of operation: controlled charge extraction (CE) mode.

The concept of a pnCCD [1] allows a direct electrical access to the pixel structure, enabling the user to modify the charge handling capacity as well as the drain off overflowing signal charges. The pnCCD is a back-illuminated direct radiation detector for ionizing radiation. It is mainly used for the detection of $\mathrm{X}$-rays, electrons and UV and visible light. It includes a fully column-parallel read out with very low noise at high frame rates. E.g. for a 264 x 264 frame store format with more than $2 \mathrm{k} \times 2 \mathrm{k}$ spatial resolution points, operated with 1,000 frames per second a read noise of a few electrons rms is achieved depending on the gain settings. This allows tracking and centroiding the signal charge cloud from a single TEM electron to less than $1 / 10$ of the pixel size. If more electrons are hitting the same pixel, the entry point from the electrons in the detector is reconstructed to a precision which is only limited by the electron statistics. This way the high flux TEM electrons can be detected with high spatial accuracy, while the diffracted low flux electrons can be recorded in the same image with high position resolution. As we operate the detector in a low noise back-illuminated mode the system is very well suited for electrons in an energy range from $2 \mathrm{keV}$ up to $300 \mathrm{keV}$ or even higher. As the electrons enter the detector through a reverse biased diode with a very shallow Boron layer on the $450 \mu \mathrm{m}$ sensitive $\mathrm{n}^{-}$ substrate, the detector is extremely radiation damage tolerant. If $1 \times 10^{15}$ electrons of $300 \mathrm{keV}$ per $\mathrm{cm}^{2}$ hit the pnCCD an increase of the readout noise from 3 electrons (rms) to only 4 electrons (rms) is observed. This allows for an uninterrupted operation for more than 10 years without visible degradation. If the noise would surpass 50 electrons (rms) the position resolution would start to degrade and a noise above 1000 electrons would start to affect the proper detection of individual $40 \mathrm{keV}$ electrons. The new modes (high charge handling capacity (HCHC) and controlled extraction) enable a whole series of new measurements in TEMs and STEMs:

(a) Mode $\mathrm{HCHC}$

High signal intensity close to primary beam and low intensity in the diffraction spots with very high spatial resolution can be recorded simultaneously. [2]. The linear response of the system is maintained. 
(b) Mode CE

(c) Mode $\mathrm{CE}+\mathrm{HCHC}$
Imaging very high signal intensities without overflowing pixels. The STEM is operated in high current mode, transients on the millisecond scale can be observed in the full frame mode or on the microsecond scale with the windowing mode.

This mode combines the high charge handling capacity and the controlled charge extraction mode. At the expense of approximately $20 \%$ less dynamic range in the linear amplitude measurement compared to the pure HCHC mode, it maintains the undistorted PSF at very high flux.

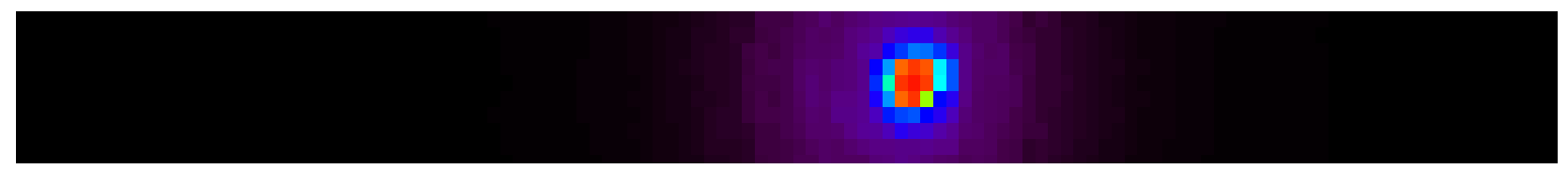

Fig 1: Zoom into the pnCCD image with $10^{9}$ signal electrons in the PSF, generated by a focussed optical laser in the pnCCD. The halo around the central spot is generated by stray light. This high signal charge density corresponds to approx. 10,000 (S)TEM electrons of $300 \mathrm{keV}$ within one PSF of the TEM within one millisecond, i.e. $10^{7}$ TEM electrons per pixel per second.
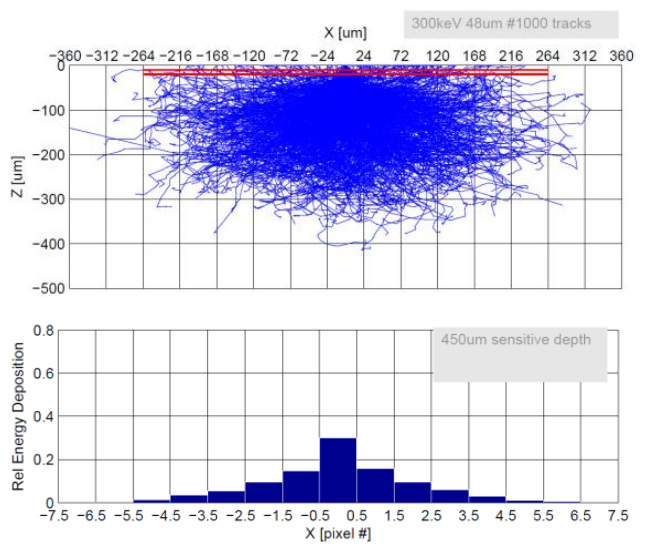

ig.2 Simulation of 1,000 electron tracks of $300 \mathrm{keV}$ electrons om a (S)TEM in a 48 umm pixel structure. They are all stopped : the $450 \mu m$ thick silicon pnCCD. The electrons generated by nization in the silicon pnCCD by the impinging electrons om the TEM are distributed along the track and may affect $\checkmark$ to 8 pixels.

Fig.3 Radially projected relative energy deposition of the 1,000 simulated electron trajectories in the $48 \mu \mathrm{m}$ pixel structure. The total amount of impinging electrons can be tetermined from the relative energy deposition, measured in the outer rim of the track distribution.

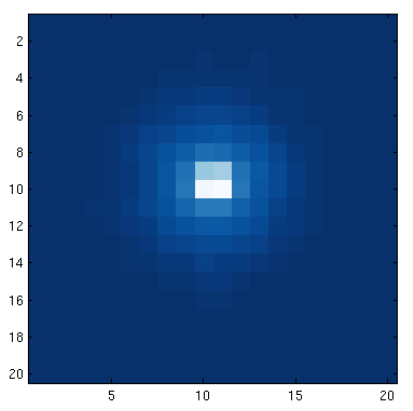

Fig.4 Measurement of 1,000 electron tracks of $300 \mathrm{keV}$ electrons from a TEM in a $48 \mu \mathrm{m}$ pixel structure. The lateral spread of the electron tracks corresponds very well to the simulations above. The CE mode takes away signal charges from the central (full) pixels while the pixels in the outer sphere remain in the linear HCHC mode of operation. The entry point of the TEM electrons in the pnCCD can be measured with subpixel resolution.

Position and time resolved X-ray spectra can be recorded simultaneously with a resolution of the order of $150 \mathrm{eV}$ to $200 \mathrm{eV}$ (FWHM) depending on gain setting and radiation background in the detector chamber. A variety of new applications in (S)TEMs is enabled, with respect to: high speed imaging, high dynamic range, single electron imaging down to $2 \mathrm{keV}$ and up to $300 \mathrm{keV}$, background rejection and imaging X-ray spectroscopy. Several applications will be shown.

Ref:

[1] L. Strüder et al., Nucl. Instr. and Meth. A 614 (2010), 483 - 496

[2] K. Müller et al, Appl. Phys. Lett. 101 (2012), p. 2121101-2121104. 